www.nature.com/jhg

\title{
HRAS mutants identified in Costello syndrome patients can induce cellular senescence: possible implications for the pathogenesis of Costello syndrome
}

\author{
Tetsuya Niihori ${ }^{1}$, Yoko Aoki ${ }^{1}$, Nobuhiko Okamoto $^{2}$, Kenji Kurosawa ${ }^{3}$, Hirofumi Ohashi ${ }^{4}$, Seiji Mizuno ${ }^{5}$, \\ Hiroshi Kawame ${ }^{6}$, Johji Inazawa ${ }^{7}$, Toshihiro Ohura ${ }^{8}$, Hiroshi Arai ${ }^{9}$, Shin Nabatame ${ }^{10}$, Kiyoshi Kikuchi ${ }^{11}$, \\ Yoshikazu Kuroki $^{12}$, Masaru Miura ${ }^{13}$, Toju Tanaka ${ }^{14}$, Akira Ohtake ${ }^{15}$, Isaku Omori ${ }^{16}$, Kenji Ihara ${ }^{17}$, \\ Hiroyo Mabe ${ }^{18}$, Kyoko Watanabe ${ }^{19}$, Shinichi Niijima ${ }^{20}$, Erika Okano ${ }^{21}$, Hironao Numabe ${ }^{22}$ and \\ Yoichi Matsubara ${ }^{1}$
}

Costello syndrome (CS) is a congenital disease that is characterized by a distinctive facial appearance, failure to thrive, mental retardation and cardiomyopathy. In 2005, we discovered that heterozygous germline mutations in HRAS caused CS. Several studies have shown that CS-associated HRAS mutations are clustered in codons 12 and 13 , and mutations in other codons have also been identified. However, a comprehensive comparison of the substitutions identified in patients with CS has not been conducted. In the current study, we identified four mutations (p.G12S, p.G12A, p.G12C and p.G12D) in 21 patients and analyzed the associated clinical manifestations of CS in these individuals. To examine functional differences among the identified mutations, we characterized a total of nine HRAS mutants, including seven distinct substitutions in codons 12 and 13, p.K117R and p.A146T. The p.A146T mutant demonstrated the weakest Raf-binding activity, and the p.K117R and p.A146T mutants had weaker effects on downstream c-Jun N-terminal kinase signaling than did codon 12 or 13 mutants. We demonstrated that these mutant HRAS proteins induced senescence when overexpressed in human fibroblasts. Oncogene-induced senescence is a cellular reaction that controls cell proliferation in response to oncogenic mutation and it has been considered one of the tumor suppression mechanisms in vivo. Our findings suggest that the HRAS mutations identified in CS are sufficient to cause oncogene-induced senescence and that cellular senescence might therefore contribute to the pathogenesis of CS. Journal of Human Genetics (2011) 56, 707-715; doi:10.1038/jhg.2011.85; published online 18 August 2011

Keywords: Costello syndrome; HRAS; phenotype-genotype; RAS/MAPK; senescence

\section{INTRODUCTION}

Costello syndrome (CS, OMIM 218040) is a genetic disorder that is characterized by a distinctive facial appearance, loose skin, failure to thrive, mental retardation, cardiomyopathy and a predisposition to tumor formation. ${ }^{1}$ Patients with CS have an estimated $13 \%$ chance of developing tumors, usually rhabdomyosarcoma, neuroblastoma or bladder cancer. ${ }^{2}$ Previously, we identified heterozygous germline HRAS mutations in patients with $\mathrm{CS}^{3}$ It has been suggested that the CS diagnosis should be applied only to patients with a mutation in HRAS because of the high risk of malignancies associated with HRAS mutations and the relative homogeneity of the CS phenotype. ${ }^{4}$

${ }^{1}$ Department of Medical Genetics, Tohoku University School of Medicine, Sendai, Japan; ${ }^{2}$ Department of Medical Genetics, Osaka Medical Center and Research Institute for Maternal and Child Health, Izumi, Japan; ${ }^{3}$ Division of Medical Genetics, Kanagawa Children's Medical Center, Yokohama, Japan; ${ }^{4}$ Division of Medical Genetics, Saitama Children's Medical Center, Saitama, Japan; ${ }^{5}$ Department of Pediatrics, Central Hospital, Aichi Human Service Center, Kasugai, Japan; ${ }^{6}$ Department of Genetic Counseling, Ochanomizu University, Tokyo, Japan; ${ }^{7}$ Department of Molecular Cytogenetics, Medical Research Institute and School of Biomedical Science, Tokyo Medical and Dental University, Tokyo, Japan; ${ }^{8}$ Division of Pediatrics, Sendai City Hospital, Sendai, Japan; ${ }^{9}$ Department of Pediatric Neurology, Morinomiya Hospital, Osaka, Japan; ${ }^{10}$ Department of Child Neurology, National Center Hospital (NCH), National Center of Neurology and Psychiatry, Tokyo, Japan; ${ }^{11}$ Department of Pediatrics, Shimane Prefectural Central Hospital, Izumo, Japan; ${ }^{12}$ Department of Neonatology, Kurashiki Central Hospital, Kurashiki, Japan; ${ }^{13}$ Division of Cardiology, Tokyo Metropolitan Children's Medical Center, Tokyo, Japan; ${ }^{14}$ Division of Clinical Genetics and Molecular Medicine, National Research Institute for Child Health and Development, Tokyo, Japan; ${ }^{15}$ Department of Pediatrics, Saitama Medical University, Moroyama, Japan; ${ }^{16}$ Department of Neonatology, Center for Maternal, Fetal \& Neonatal Medicine, Tokyo Metropolitan Bokutoh Hospital, Tokyo, Japan; ${ }^{17}$ Department of Pediatrics, Graduate School of Medical Sciences, Kyushu University, Fukuoka, Japan; ${ }^{18}$ Department of Child Development, Faculty of Life Sciences, Kumamoto University, Kumamoto, Japan; ${ }^{19}$ Division of Pediatrics, National Hospital Organization Kokura Medical Center, Kitakyushu, Japan; ${ }^{20}$ Department of Pediatrics, Juntendo University, Nerima Hospital, Tokyo, Japan; ${ }^{21}$ Department of Pediatrics, Jikei University School of Medicine, Tokyo, Japan and ${ }^{22}$ Department of Clinical Genetics, Kyoto University Hospital, Kyoto, Japan

Correspondence: Dr T Niihori or Dr Y Aoki, Department of Medical Genetics, Tohoku University School of Medicine, 1-1 Seiryo-machi, Sendai 980-8574, Japan. E-mail: tniihori@med.tohoku.ac.jp or aokiy@med.tohoku.ac.jp

Received 4 April 2011; revised and accepted 15 June 2011; published online 18 August 2011 
A total of 14 HRAS missense mutations and one duplication mutation have been reported in 185 patients with $\mathrm{CS}^{3,5-23}$ or congenital myopathy with excess of muscle spindles. ${ }^{24}$ Most of these mutations have previously been reported as somatic and oncogenic mutations in various tumors. More than $90 \%$ of the mutations found in CS patients are clustered in codons 12 and 13 (p.G12A/S/V/C/D/E and p.G13C/D). Other mutations, including p.Q22K, p.E37dup, p.T58I, p.E63K, p.K117R, p.A146V and p.A146T, have also been identified, albeit rarely. Although the clinical manifestations of CS appear to be homogeneous, several genotype-phenotype correlations have been reported. Previous studies have also suggested that CS patients with the p.G12A mutation may have an increased risk of malignancy, compared with patients with p.G12S. ${ }^{7}$ Patients with the p.G12C mutation had a more severe CS phenotype; these individuals developed severe hypertrophic cardiomyopathy and died in the neonatal period. Patients with p.K117R or p.A146V had a milder and more unusual CS phenotype, compared with patients with mutations in codon 12 or 13. Though detailed analyses of some mutants have been performed, ${ }^{13,25-28}$ a comprehensive comparison of the substitutions identified in patients with CS has not been conducted.

The activated RAS/mitogen-activated protein kinase (MAPK) pathway generally stimulates cell proliferation, but it can also result in antiproliferation under certain conditions. Overexpressing HRAS p.G12V in human and murine fibroblasts caused oncogene-induced senescence (OIS), ${ }^{29-31}$ which protects cells from proliferating in the presence of oncogene-induced damage. ${ }^{32,33}$ OIS is a cellular reaction that controls cell proliferation in response to oncogenic mutation and is considered a tumor suppression mechanism in vivo. ${ }^{34,35}$ Studies of a zebrafish model of CS, which expresses HRAS p.G12V, have shown that progenitor cells in the adult heart and brain undergo cellular senescence, suggesting that OIS in adult progenitor cells contributes to the development of CS. We hypothesized that OIS would be a key mechanism of the clinical manifestations in patients with CS, including short stature, osteoporosis and tumor suppressive effects. However, it has not been verified that HRAS mutants other than p.G12V cause cellular senescence.

The three aims of this study were the following: (1) to examine the detailed clinical manifestations of CS in patients with HRAS mutations, (2) to characterize a large panel of HRAS mutants to look for differences among various mutations located in codon $12 / 13$ and to compare the effects of mutants in codon 12/13 with those of p.K117R/ p.A146T, and (3) to clarify whether HRAS mutants other than p.G12V can cause OIS. To address these issues, we analyzed the HRAS mutations in CS patients and studied the Raf-binding activity, downstream signaling and ability to cause senescence of a large panel of HRAS mutants.

\section{MATERIALS AND METHODS}

\section{Patients}

A total of 31 patients suspected of having CS were recruited to the study. The diagnosis of CS was evaluated by clinical geneticists. All patients had sporadic cases. The study was approved by the Ethics Committee of the Tohoku University School of Medicine.

\section{Mutation analysis}

We sequenced the HRAS genes of all patients in the study to confirm the diagnosis of CS. After obtaining written informed consent, genomic DNA was isolated from the peripheral leukocytes of patients. Four coding exons of HRAS from 31 CS patients were sequenced. Each HRAS exon with flanking intronic sequences was amplified using primers based on sequences obtained from GenBank (GenBank accession no. NT035113). The M13 reverse or forward sequence was added to the $5^{\prime}$ end of the polymerase chain reaction primers for use, as a sequencing. polymerase chain reaction was performed in a $30 \mu \mathrm{l}$ reaction containing $10 \mathrm{~mm}$ Tris- $\mathrm{HCl}(\mathrm{pH} 8.3), 50 \mathrm{~mm} \mathrm{KCl}, 1.5 \mathrm{mM} \mathrm{MgCl}_{2}$, $0.2 \mathrm{~mm}$ deoxyribonucleotide triphosphate, $10 \%(\mathrm{v} / \mathrm{v})$ dimethyl sulfoxide, 0.4 pmol each primer, $100 \mathrm{ng}$ genomic DNA and 2.5 units of Taq DNA polymerase. The reaction consisted of 35 cycles of denaturation at $94{ }^{\circ} \mathrm{C}$ for $15 \mathrm{~s}$, annealing at $57^{\circ} \mathrm{C}$ for $15 \mathrm{~s}$ and extension at $72^{\circ} \mathrm{C}$ for $30 \mathrm{~s}$. The products were gel-purified and sequenced on an Applied Biosystems 3130 Genetic Analyzer (Applied Biosystems, Foster City, CA, USA).

\section{Plasmids}

To introduce exogenous wild-type or mutated HRAS into cultured cells, we constructed plasmids encoding wild-type or mutant HRAS cDNAs. Human HRAS cDNA in pUSEamp was purchased from Upstate Biotechnology (Lake Placid, NY, USA). The plasmid was digested with EcoRI and subcloned into pBluescript KSII+ (Stratagene, La Jolla, CA, USA). Substitutions generating p.G12V (c.35G>T), p.G12A (c.35G >C), p.G12S (c.34G>A), p.G12C (c.34G > C), p.G12D (c.35G>A), p.G13C (c.37G>C), p.G13D (c.38G>A), p.K117R $(c .350 \mathrm{~A}>\mathrm{G})$ or p.A146T (c.436G $>$ A) were introduced using the QuikChange Site-Directed mutagenesis kit (Stratagene). All mutant and wildtype constructs were verified by sequencing. The full-length wild-type and mutant HRAS cDNAs were digested with EcoRI and subcloned into the pBabe-puro retroviral vector (GenHunter, Nashville, TN, USA) and the pCAGGS expression vector (gifted by Dr Jun-ichi Miyazaki of Osaka University). The pBabe-zeo-Ecotropic Receptor plasmid (Addgene plasmid 10687, Addgene Inc., Cambridge, MA, USA) was obtained from Addgene.

\section{Cell culture and senescence-associated $\beta$-galactosidase staining}

NIH 3T3 cells, human fibroblast BJ cells and the Phoenix Ampho and Eco packaging cell lines were purchased from the American Tissue Culture Collection (Manassas, VA, USA). NIH 3T3 cells were maintained in Dulbecco's modified Eagle medium containing $10 \%$ calf serum, $100 \mathrm{U} / \mathrm{ml}$ penicillin and $100 \mu \mathrm{g} / \mathrm{ml}$ streptomycin. BJ and Phoenix cells were maintained in Dulbecco's modified Eagle medium containing $10 \%$ fetal calf serum, $100 \mathrm{U} / \mathrm{ml}$ penicillin and $100 \mu \mathrm{g} / \mathrm{ml}$ streptomycin. To characterize the phenotypes of cells overexpressing wild-type or mutated HRAS, senescence associated $\beta$-galactosidase staining was performed with the Senescence $\beta$-Galactosidase Staining Kit (Cell Signaling Technology, Beverly, MA, USA) according to the manufacturer's protocol.

\section{Ras activation assay}

We performed RAS activation assays to clarify the functional differences among the HRAS mutants identified in patients with CS. The Ras activation assay kit was purchased from Millipore (Billerica, MA, USA). NIH 3T3 cells were plated in 6-well plates at $1.5 \times 10^{5}$ cells per well. Cells were transfected using Lipofectamine Plus (Invitrogen, Carlsbad, CA, USA) with $1 \mu \mathrm{g}$ wild-type or mutant HRAS construct. The assay was performed according to the manufacturer's protocol.

\section{Luciferase assay}

We used luciferase assays to examine the effect of the identified mutations on the RAS pathway. NIH 3T3 cells were plated in 12 -well plates at $1 \times 10^{5}$ cells per well. After $24 \mathrm{~h}$, cells were transiently transfected with $700 \mathrm{ng}$ pFR-luc, $10 \mathrm{ng}$ pFA2-Elk1 or $10 \mathrm{ng}$ pFA2-cJun, $7 \mathrm{ng}$ phRLnull-luc and $35 \mathrm{ng}$ wild-type or mutant HRAS construct, using Lipofectamine Plus (Invitrogen). At $18 \mathrm{~h}$ after transfection, the cells were serum starved in Dulbecco's modified Eagle medium for $24 \mathrm{~h}$. Cells were then harvested in passive lysis buffer, and luciferase activity was assayed using the Promega Dual-Luciferase assay kit (Promega, Madison, WI, USA). Renilla luciferase expressed by phRLnull-luc was used to normalize the transfection efficiency. The experiments were performed in triplicate. Statistical analysis was performed with Tukey's multiple comparison test.

\section{Western blotting}

We performed western blotting against molecular markers of premature senescence to confirm their expression in cells overexpressing HRAS. Cells were harvested at the indicated times, washed in ice-cold phosphate-buffered saline and lysed on ice in lysis buffer (10 mm Tris- $\mathrm{HCl}, \mathrm{pH} 7.5$ and $1 \%$ sodium 
dodecyl sulfate). Lysates were boiled for $5 \mathrm{~min}$ and centrifuged at $13000 \mathrm{~g}$ for $10 \mathrm{~min}$ at $4{ }^{\circ} \mathrm{C}$. Protein concentrations were estimated using the Lowry or Bradford method (BioRad, Hercules, CA, USA), and each lysate was adjusted to equalize the protein concentrations. Equal volumes of lysates were mixed with $2 \times$ sodium dodecyl sulfate sample buffer and boiled for $5 \mathrm{~min}$. Electrophoresis was performed on 5-15\% sodium dodecyl sulfate-polyacrylamide gels. After separation, proteins were transferred to nitrocellulose membranes. The membranes were blocked in 5\% non-fat dry milk in Tris-buffered saline with $0.1 \%$ Tween 20 for $1 \mathrm{~h}$ at room temperature and incubated overnight at $4{ }^{\circ} \mathrm{C}$ with one of the following primary antibodies: HRAS (sc-520, Santa Cruz Biotechnology, Santa Cruz, CA, USA), phospho-p44/42MAPK, p44/42MAPK (\#9102 and \#9101, respectively, Cell Signaling Technology, Danvers, MA, USA), p16 (sc-468, Santa Cruz Biotechnology), phospho-p53 (Ser15) (\#9284, Cell Signaling Technology) or $\beta$-actin (A5316, Sigma, St. Louis, MO, USA). Detection was performed using the enhanced chemiluminescence method (Amersham, GE Healthcare UK, Amersham, UK), with the appropriate peroxidase-conjugated secondary antibody.

\section{Retroviral gene transfer}

We generated cells that stably overexpressed wild-type or mutant HRAS by retroviral gene transfer. Phoenix cells $\left(5 \times 10^{6}\right)$ were plated in a $10 \mathrm{~cm}$ dish, incubated for $24 \mathrm{~h}$ and then transfected with $18 \mu \mathrm{g}$ of retroviral plasmid using Fugene6 (Roche Applied Science, Mannheim, Germany). After $48 \mathrm{~h}$, the viruscontaining medium was filtered through a $0.45-\mu \mathrm{m}$ filter and supplemented with $4 \mu \mathrm{g} / \mathrm{ml}$ polybrene (Sigma) to collect the virus (first supernatant). Viruses were collected after an additional $24 \mathrm{~h}$ as before (second supernatant). BJ fibroblasts were plated at $6 \times 10^{5}$ cells per $10 \mathrm{~cm}$ dish and incubated overnight. For infections, the culture medium was replaced with the first viral supernatant and incubated at $37^{\circ} \mathrm{C}$ for $8 \mathrm{~h}$, after which the second viral supernatant was added. Infected cell populations were selected $40 \mathrm{~h}$ later, using $2 \mu \mathrm{g} / \mathrm{ml}$ puromycin or $200 \mu \mathrm{g} / \mathrm{ml}$ zeocin. The ecotropic retrovirus receptor was introduced into the BJ human fibroblasts by infecting cell populations with an amphotropic vector (pBabe-zeo-ecotropic receptor produced in Phoenix Ampho cells), allowing subsequent infection with ecotropic viruses.

\section{RESULTS}

\section{Mutation analysis in patients with CS}

Genomic sequencing analysis of 32 individuals with confirmed or suspected CS revealed four different missense mutations in 21 patients: a heterozygous $34 \mathrm{G}>\mathrm{A}$ mutation (p.G12S) in 16 patients, a heterozygous $35 \mathrm{G}>\mathrm{C}$ mutation (p.G12A) in three patients, a heterozygous $34 \mathrm{G}>\mathrm{T}$ change (p.G12C) in one patient, and a $35 \mathrm{G}>\mathrm{A}$ change (p.G12D) in one patient.

The clinical data for 21 CS mutation-positive patients are shown in Table 1. Curly and/or sparse hair (21/21), failure to thrive (21/21), coarse facial appearance (20/20), deep palmar/planter creases $(20 / 21)$, soft, loose skin (18/21) and relative macrocephaly (17/21) were observed at high frequency in patients with CS, as previously reported. ${ }^{1,3}$ Laryngomalacia (soft larynx), which has been reported in several patients with $\mathrm{CS},{ }^{36-38}$ was observed in three patients. One patient had hypertension, which was also observed in a mouse model of $\mathrm{CS}^{39}$ One patient had glycogen storage disease type III, as previously reported by Kaji et al., 40 accompanied by a p.G12S mutation. Bladder cancer was observed in one patient.

One patient (NS 223) with HRAS p.G12C had severe clinical manifestations of CS and was treated with pravastatin. ${ }^{41}$ She was born at 23 weeks of gestation with extremely low birth weight ( $766 \mathrm{~g}$, $>90$ th percentile), even though her mother had received tocolytic therapy. Her Apgar scores were 3 and 7 at 1 and $5 \mathrm{~min}$, respectively. She required mechanical ventilation. Extubation was attempted periodically beginning at day 70 , but it was unsuccessful until she turned 2 years old, because of her laryngomalacia and increased mucus secretion. Hypertrophic cardiomyopathy was first observed on day 38. The patient was given propranolol and cibenzoline to control the gradual progression of hypertrophic cardiomyopathy. Cardiac arrest after extubation occurred on day 192 and the patient was successfully resuscitated. Papillomas developed at approximately 11 months of age. Erosion and itching of skin were not well controlled by topical steroids or antihistamines. Pravastatin $(0.2 \sim 0.4 \mathrm{mg} / \mathrm{kg} / \mathrm{day})$ was administered in anticipation of its suppressive effect on RAS, beginning when she was 16 months old. Thereafter, the papillomas disappeared once and appeared again, but were less numerous than when they first appeared. The effects of pravastatin on hypertrophic cardiomyopathy were not obvious. The patient was discharged from the hospital at 2 years of age.

\section{Analysis of mutant HRAS activation states and effects on the downstream pathway}

We performed RAS activation assays to elucidate functional differences among the mutants identified in patients with CS. We transfected NIH 3T3 cells with wild-type HRAS or one of the nine HRAS mutants identified in patients with CS. We found an increase in guanosine triphosphate (GTP)-bound HRAS in all cells transfected with HRAS p.G12V, p.G12A, p.G12S, p.G12C, p.G12D, p.G13C, p.G13D, p.K117R and p.A146T. We did not detect any differences among the increases of GTP-bound HRAS in the cells transfected with HRAS p.G12V, p.G12A, p.G12S, p.G12C, p.G12D, p.G13C, p.G13D and p.K117R. The increase in the level of GTP-bound HRAS-p.A146T was milder than that of other mutants.

Next, we examined the effect of the identified mutations on the RAS pathway by studying the activation of ELK1 and c-Jun in transfected $\mathrm{NIH}$ 3T3 cells. ELK1 and c-Jun are the main nuclear targets of extracellular signal-regulated kinase and c-Jun N-terminal kinase, respectively. We transfected the pFR-luc trans-reporter vector, the pFA2-ELK1 or pFA2-cJun vector and the phRLnull-luc vector into NIH 3 T3 cells and determined the relative luciferase activity (RLA) in each cell line. The basal RLA in cells transfected with active MEK1 or MEKK constructs showed a three-fold increase, compared with cells transfected with wild-type HRAS cDNA (Figure 1a). A significant increase in RLA was observed upon transfection with ELK1 and HRAS p.G12V, p.G12A, p.G12S, p.G12C, p.G12D, p.G13C, p.G13D, p.K117R and p.A146T (Figure 1b). The RLA of c-Jun was significantly increased in cells transfected with HRAS p.G12V, p.G12A, p.G12S, p.G12C, p.G12D, p.G13C and p.G13D (Figure 1c). In these assays with ELK1 and c-Jun, we observed no significant difference among RLAs in the cells transfected with HRAS p.G12V, p.G12A, p.G12S, p.G12C, p.G12D, p.G13C and p.G13D. These results suggest that HRASp.K117R and p.A146T had a weaker effect on the c-Jun N-terminal kinase pathway than the other mutants.

\section{Cellular senescence in human fibroblasts transfected with HRAS mutants}

The HRAS p.G12V mutant causes a senescence phenotype when transduced into human diploid fibroblasts. To examine the ability of the various mutants identified in patients with CS to cause senescence, we introduced wild-type or mutated HRAS cDNAs into human fibroblast BJ cells, using retroviral gene transfer. Figure 2a shows these cells six days after infection. Wild-type HRAS-induced cells exhibited a narrow and elongated morphology and they were not flat like senescent cells. They proliferated at levels similar to cells transfected with empty vector. In contrast, the p.G12V, p.G12A, p.G12S, p.G12C, p.G12D, p.G13C, p.G13D, p.K117R and p.A146T mutants produced cells with a senescence phenotype, exhibiting flat, enlarged and multivacuolated morphology and prominent nucleoli. Senescence 
neg

HRAS mutants in CS and senescence

T Niihori et al

710

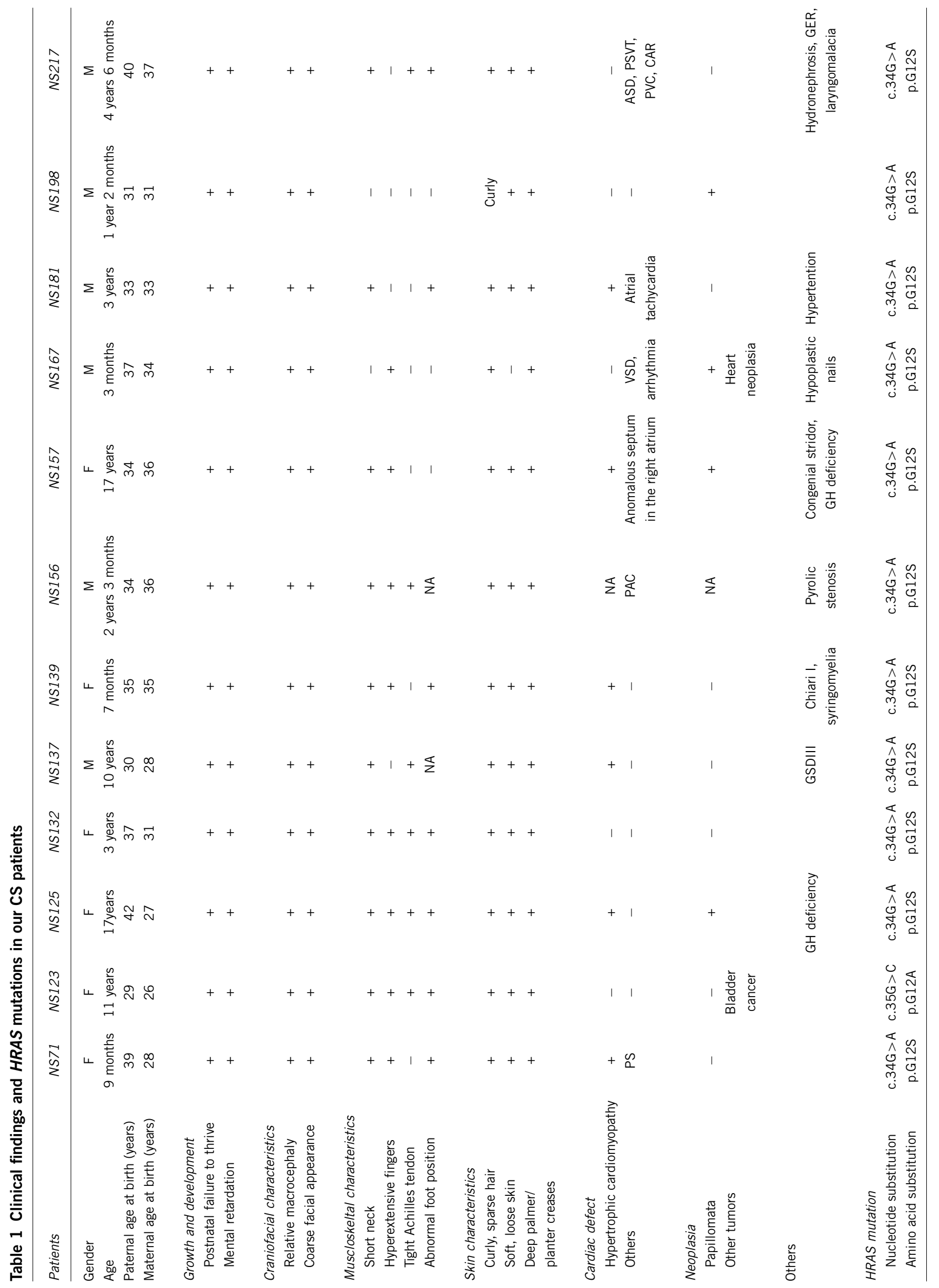

Journal of Human Genetics 


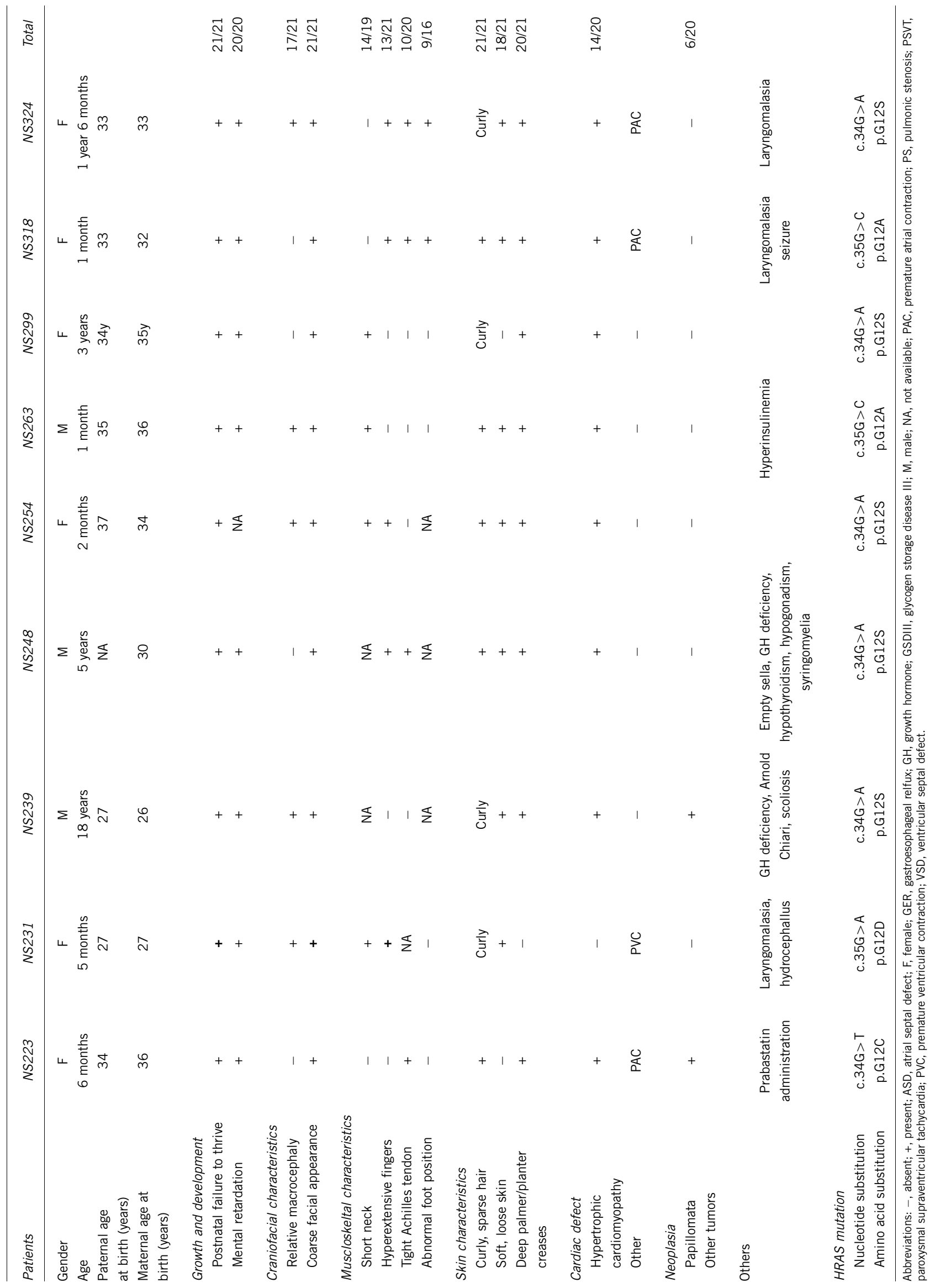




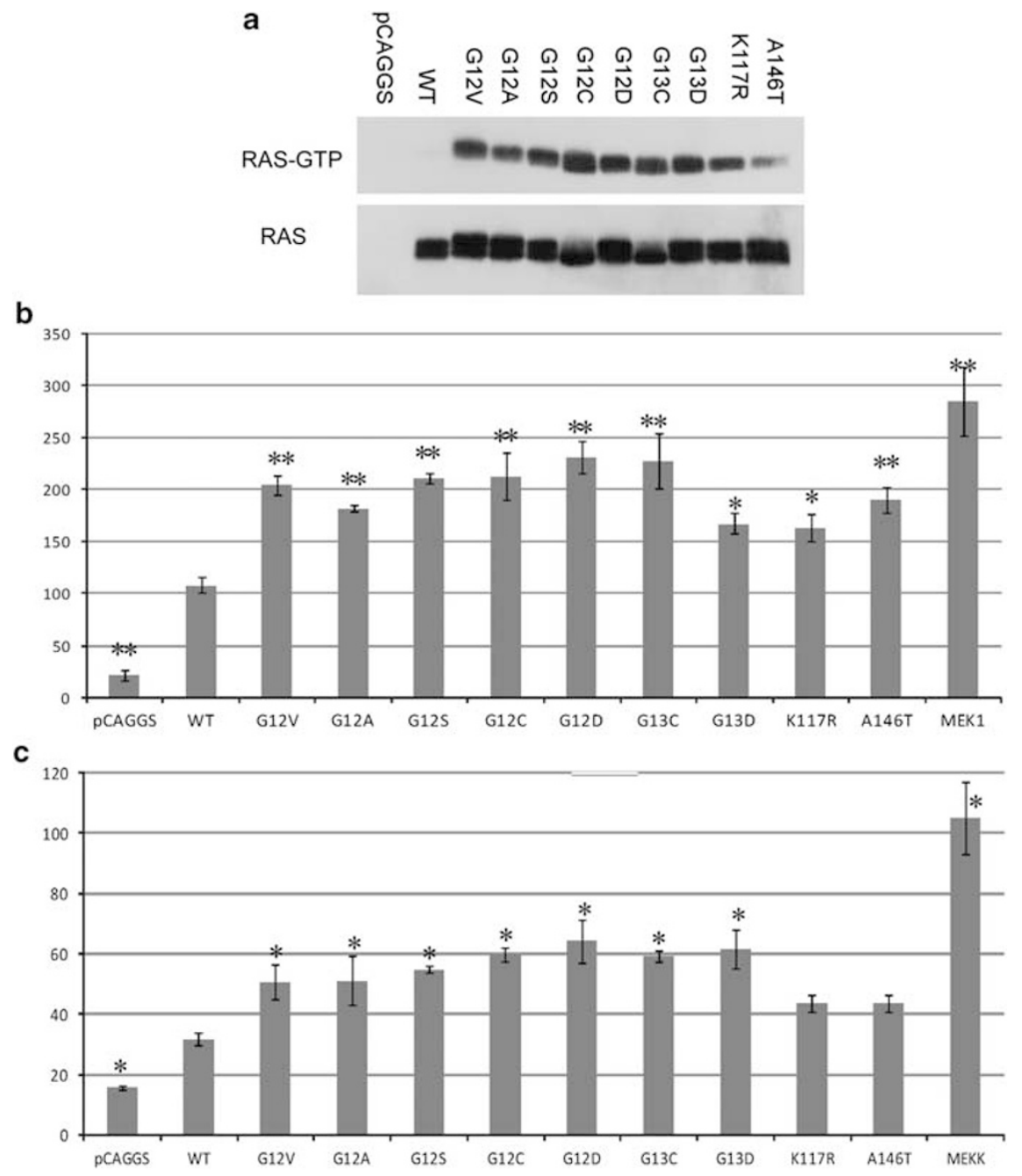

Figure 1 Functional characterization of HRAS mutants. (a) Ras-guanosine triphosphate (GTP) in NIH 3T3 cells transfected with wild-type or mutant HRAS constructs. HRAS protein levels were similar in NIH3T3 cells expressing each protein and were subsequently used as a loading control. (b, c) Stimulation of ELK (b) and c-Jun (c) transcription by HRAS mutants. The ELK-and c-Jun-GAL4 vectors and GAL4-luciferase trans-reporter vector were transiently co-transfected with various HRAS constructs into unstimulated NIH 3T3 cells. Relative luciferase activity (RLA) was normalized to the activity of a co-transfected control vector (phRLnull-luc) expressing Renilla reniformis luciferase. The results are expressed as the means and s.d. from triplicate samples. MEK1 and MEKK were used as positive controls. WT, wild type. ${ }^{*} P<0.05 ;{ }^{*} P<0.01$ compared with WT.

associated $\beta$-galactosidase staining confirmed that these cells showed cellular senescence.

Two downstream signaling pathways, p53 and Rb-p16, are activated during cellular senescence. To examine oncogene induced cellular senescence at the molecular level, we assessed senescence markers, including phosphorylated extracellular signal-regulated kinase, phosphorylated p53 and p16, in cells expressing HRAS mutant proteins (Figure 2b). As expected, phosphorylated p53 (Ser15) and p16 levels, as well as phospho-extracellular signal-regulated kinase levels, were significantly increased in the cells transfected with HRAS mutants relative to cells transfected with mock vector or wild-type HRAS. These results demonstrate that not only p.G12V, but also the other eight CS-related HRAS mutants, can cause OIS.

\section{DISCUSSION}

In this study, we identified four HRAS mutations in 21 patients with CS and evaluated their detailed clinical manifestations of the disease in these patients. Biochemical analyses, including a GTP binding assay and luciferase assays to detect ELK and c-Jun trans-activation, showed that there were no significant differences among the analyzed mutations in codon 12/13. The p.A146T mutant demonstrated the weakest Raf binding activity, and the p.K117R and p.A146T mutants had weaker effects on downstream c-Jun N-terminal kinase signaling than mutants in codon 12 or 13 . Our results indicated that all HRAS mutants detected in CS patients were able to cause OIS.

Our study is the first to demonstrate that HRAS mutants other than p.G12V can induce senescence when they are overexpressed in human fibroblasts. The symptoms of CS seem to be caused by either hyperproliferation or hypoproliferation, coupled with growth factor resistance, which may be ascribable to DNA damage response or OIS. Postnatal cerebellar tonsillar herniation, Chiari 1 malformation, ${ }^{42}$ deep palmar and plantar creases and papillomata may all be caused by hyperproliferation. In contrast, the poor weight gain, short stature and endocrine dysfunction observed in CS patients ${ }^{43-45}$ might be caused by hypoproliferation. Adult brain and heart progenitor cells in a zebrafish CS model with a homozygous HRAS p.G12V mutation 


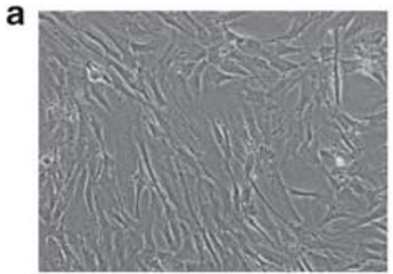

pBabe

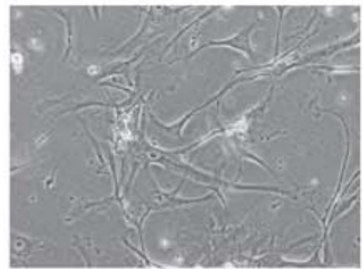

G12A

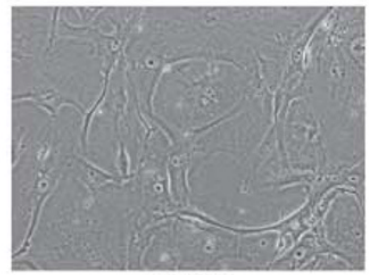

G12D

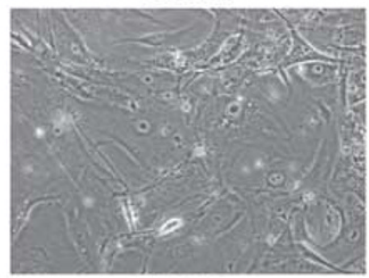

K117R

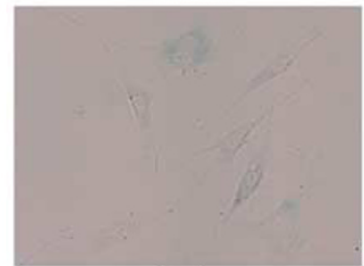

pBabe

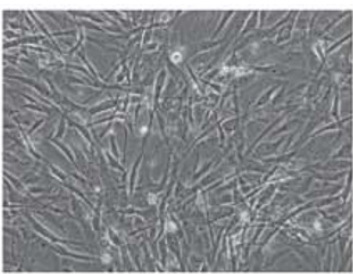

WT

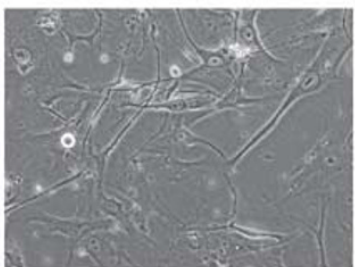

G12S

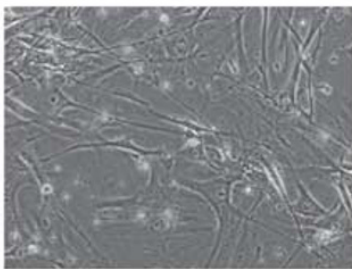

G13C

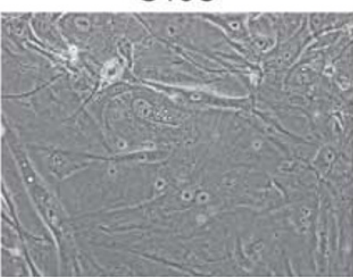

A146T

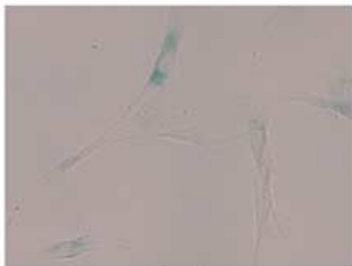

WT

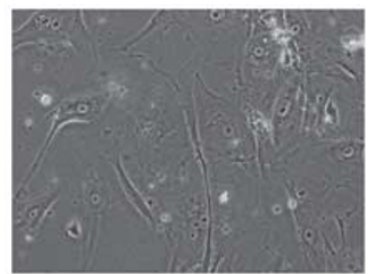

G12V

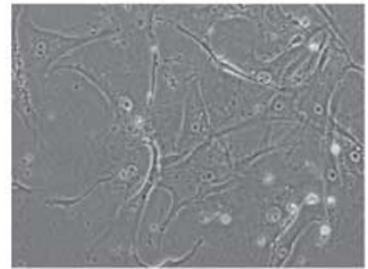

G12C

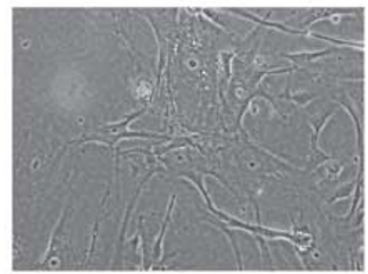

G13D

b

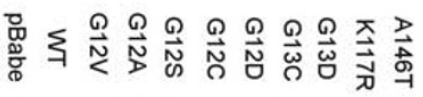

Phospho-p53(Ser15)

p16

HRAS

Phospho-ERK

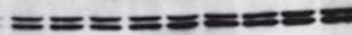

ERK

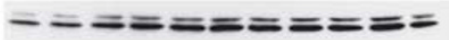

$\beta$-actin

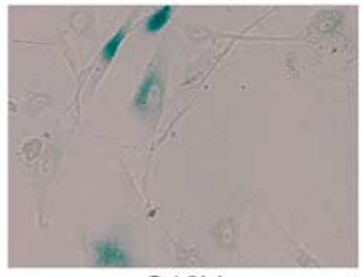

G12V

Figure 2 Effect of Costello syndrome (CS)-associated HRAS mutants on primary fibroblasts. (a) BJ cells transduced with retroviruses expressing wild-type or mutant HRAS. Images in the lowest tier show senescence-associated $\beta$-galactosidase staining. (b) Immunoblots of cellular lysates from BJ cells transduced with empty vector (pBabe) or with wild-type or mutant HRAS retroviruses. 
exhibited cellular senescence, suggesting that the age-related worsening of the Costello phenotype ${ }^{46}$ might occur, because the replicative capability of adult progenitor cells is exhausted. Osteoporosis has frequently been found in adult patients with $\mathrm{CS},{ }^{47}$ suggesting that cellular senescence affects osteogenesis. However, further studies will be needed to determine whether OIS indeed contributes to the pathogenesis in patients with CS.

It has been suggested that clinical symptoms vary among patients with mutations in codon 12 or 13 . In previous studies, a total of 19 CS patients have been reported to die from severe cardiomyopathy, cardiac arrhysmia, rhabdomyosarcoma, respiratory failure, multiorgan failure or sepsis. The number of fatal cases was $5 / 138$ patients with p.G12S, 4/6 with p.G12C, 3/17 with p.G12A, 3/4 with p.G12D, 2/2 with p.G12V, 1/1 with p.G12E and $1 / 1$ with p.E63K. ${ }^{3,5-23}$ The mortality of patients with p.G12C or p.G12D was significantly higher than that of the patients with the more common p.G12S ( $P=0.026$ by Fisher's exact test). Previous studies have shown that the p.G12V substitution has the highest transformative potential (p.G12V>p.G12A, p.G12S, p.G12C, p.G12D > p.G13D) and is the most frequently found mutation in human tumors. ${ }^{48,49}$ However, our Ras activity assays and luciferase assays did not show any differences among HRAS codon 12/13 mutants. This may be due to the extremely high expression level of HRAS protein in our transient transfection study, which could make it difficult to detect subtle differences between mutants. Further studies will be necessary to clarify whether the high mortality in patients with p.G12C or p.G12D is due to functional differences in these mutants or due to bias because of our small sample size of patients.

Mutations at codons 117 and 146 are rare in CS and somatic cancers. Meanwhile, mutations at codons G12, G13 and Q61 have been shown to impair intrinsic and GTPase activating proteinmediated GTP hydrolysis, leading to elevated levels of cellular RASGTP. It has been reported that the nucleotide exchange rate of both p.K117R and p.A146V HRAS is increased, relative to wild type. ${ }^{13,27,28}$ However, the transformational potential of p.A146V HRAS is partially activated ${ }^{27}$ whereas that of p.K117R-HRAS is not; its transformational activity is instead similar to that of GTPase impaired mutants. ${ }^{28}$ Our results and those of other reports suggest that p.K117R and p.A146T have milder effects on downstream effectors than do mutations in codon 12/13.

The clinical manifestations of CS in patients with p.K117R or p.A146V mutations suggest that these alleles have distinct effects, compared with mutations in codon 12/13. Of two CS patients with a p.K117R mutation, one patient had an atypical phenotype such as microretrognatism and slightly less-pronounced plantar and palmar creases. $^{7}$ The other patient had mild craniofacial manifestations of CS. ${ }^{13}$ One patient with the p.A146V mutation showed a mildly coarse face and did not have deep palmar creases. ${ }^{6}$ These atypical phenotypes might be attributed to the mild effects of p.K117R or p.A146V compared with codon 12/13 mutants.

Inhibitors of the RAS/MAPK pathway could provide benefits for patients with RAS/MAPK syndromes. Statins are 3-hydroxy-3-methylglutaryl-CoA reductase inhibitors that result in decreased isoprenylation of $\mathrm{RAS}^{50}$ and are now widely used for the treatment of hyperlipidemia. Statins have been used to modify the clinical manifestation of neurofibromatosis type I, which is caused by a genetic defect in a negative regulator of the RAS/MAPK pathway. Studies using mouse models of NF1 (Nf1 mice) have shown that treatment with a statin reverses the cognitive deficits of these mice. ${ }^{51}$ A randomized control trial for neurofibromatosis type I treatment with simvastatin had a negative outcome. ${ }^{52}$ Furthermore, statins have displayed antitumor activity in experimental tumor models, though clinical antitumor effects of statins have not been established. ${ }^{53}$ Welldesigned clinical studies will be needed to determine the effects of statins or other RAS inhibitors on manifestations of CS.

In conclusion, we identified HRAS mutations in 21 patients and examined the clinical manifestations of mutation-positive patients. Functional analysis revealed that CS-causing mutant HRAS proteins caused OIS in human fibroblasts. These findings may help enable more accurate prognoses for patients with HRAS mutations and contribute to our understanding of the mechanism underlying CS pathogenesis.

\section{CONFLICT OF INTEREST}

The authors declare no conflict of interest.

\section{ACKNOWLEDGEMENTS}

We thank the patients who participated in this study and their families and doctors, including Naoki Watanabe and Tomohiro Iwasaki, who referred the cases. We are grateful to Dr Garry Nolan of Stanford University for supplying Phoenix-Eco and Ampho cells, to Dr William C Hahn for supplying the pBabe-zeo-ecotropic receptor vector, and to Dr Jun-ichi Miyazaki of Osaka University for supplying the pCAGGS expression vector. We are also grateful to Drs Noriko Ishida and Keiko Nakayama for their technical assistance with the infection of retroviral vectors. We thank Kumi Kato and Hasumi Haba for their technical assistance. This work was supported by Grants-in-Aids for young scientists (A and S) from the Ministry of Education, Culture, Sports, Science and Technology of Japan (nos. 19689022, 21689029 and 19679005) to TN and YA, the Science and Technology Foundation of Japan Grant-in-Aid for Scientific Research to TN, and the Ministry of Health, Labour and Welfare to YM and YA.

1 Hennekam, R. C. Costello syndrome: an overview. Am. J. Med. Genet. C. Semin. Med. Genet. 117C, 42-48 (2003).

2 Aoki, Y., Niihori, T., Narumi, Y., Kure, S. \& Matsubara, Y. The RAS/MAPK syndromes: novel roles of the RAS pathway in human genetic disorders. Hum. Mutat. 29, 992-1006 (2008)

3 Aoki, Y., Niihori, T., Kawame, H., Kurosawa, K., Ohashi, H., Tanakam, Y. et al. Germline mutations in HRAS proto-oncogene cause Costello syndrome. Nat. Genet. 37, 1038-1040 (2005).

4 Kerr, B., Allanson, J., Delrue, M. A., Gripp, K. W., Lacombe, D., Lin, A. E. et al. The diagnosis of Costello syndrome: nomenclature in Ras/MAPK pathway disorders. Am. J. Med. Genet. A 146A, 1218-1220 (2008).

5 Estep, A. L., Tidyman, W. E., Teitell, M. A., Cotter, P. D. \& Rauen, K. A. HRAS mutations in Costello syndrome: detection of constitutional activating mutations in codon 12 and 13 and loss of wild-type allele in malignancy. Am. J. Med. Genet. A. 140, 8-16 (2006).

6 Gripp, K. W., Lin, A. E., Stabley, D. L., Nicholson, L., Scott, C. I. Jr, Doyle, D. et al. HRAS mutation analysis in Costello syndrome: genotype and phenotype correlation. Am. J. Med. Genet. A. 140, 1-7 (2006).

7 Kerr, B., Delrue, M. A., Sigaudy, S., Perveen, R., Marche, M., Burgelin, I. et al. Genotype-phenotype correlation in Costello syndrome: HRAS mutation analysis in 43 cases. J. Med. Genet. 43, 401-405 (2006)

8 van Steensel, M. A., Vreeburg, M., Peels, C., van Ravenswaaij-Arts, C. M., Bijlsma, E., Schrander-Stumpel, C. T. et al. Recurring HRAS mutation G12S in Dutch patients with Costello syndrome. Exp. Dermatol. 15, 731-734 (2006).

9 Gripp, K. W., Lin, A. E., Nicholson, L., Allen, W., Cramer, A., Jones, K. L. et al. Further delineation of the phenotype resulting from BRAF or MEK1 germline mutations helps differentiate cardio-facio-cutaneous syndrome from Costello syndrome. Am. J. Med. Genet. A. 143A, 1472-1480 (2007)

10 Orstavik, K. H., Tangeraas, T., Molven, A. \& Prescott, T. E. Distal phalangeal creases-a distinctive dysmorphic feature in disorders of the RAS signalling pathway? Eur. J. Med. Genet. 50, 155-158 (2007).

11 Sovik, O., Schubbert, S., Houge, G., Steine, S. J., Norgard, G., Engelsen, B. et al. De novo HRAS and KRAS mutations in two siblings with short stature and neurocardio-facio-cutaneous features. J. Med. Genet. 44, e84 (2007).

12 Zampino, G., Pantaleoni, F., Carta, C., Cobellis, G., Vasta, I., Neri, C. et al. Diversity parental germline origin, and phenotypic spectrum of de novo HRAS missense changes in Costello syndrome. Hum. Mutat. 28, 265-272 (2007).

13 Denayer, E., Parret, A., Chmara, M., Schubbert, S., Vogels, A., Devriendt, K. et al. Mutation analysis in Costello syndrome: functional and structural characterization of the HRAS pLys117Arg mutation. Hum. Mutat. 29, 232-239 (2008). 
14 Gripp, K. W., Innes, A. M., Axelrad, M. E., Gillan, T. L., Parboosingh, J. S., Davies, C. et al. Costello syndrome associated with novel germline HRAS mutations: an attenuated phenotype? Am. J. Med. Genet. A. 146A, 683-690 (2008).

$15 \mathrm{Hou}, \mathrm{J}$. W. Rapidly progressive scoliosis after successful treatment for osteopenia in Costello syndrome. Am. J. Med. Genet. A. 146, 393-396 (2008).

16 Limongelli, G., Pacileo, G., Digilio, M. C., Calabro, P., Di Salvo, G., Rea, A. et al Severe, obstructive biventricular hypertrophy in a patient with Costello syndrome: clinical impact and management. Int. J. Cardiol. 130, e108-e110 (2008).

17 Schulz, A.L., Albrecht, B., Arici, C., van der Burgt, I., Buske, A., Gillessen-Kaesbach, G. et al. Mutation and phenotypic spectrum in patients with cardio-facio-cutaneous and Costello syndrome. Clin. Genet. 73, 62-70 (2008).

18 Gremer, L., De Luca, A., Merbitz-Zahradnik, T., Dallapiccola, B., Morlot, S., Tartaglia, M. et al. Duplication of Glu37 in the switch I region of HRAS impairs effector/GAP binding and underlies Costello syndrome by promoting enhanced growth factordependent MAPK and AKT activation. Hum. Mol. Genet. 19, 790-802 (2010).

19 Kuniba, H., Pooh, R.K., Sasaki, K., Shimokawa, O., Harada, N., Kondoh, T. et al. Prenatal diagnosis of Costello syndrome using 3D ultrasonography amniocentesis confirmation of the rare HRAS mutation G12D. Am. J. Med. Genet. A. 149A, 785787 (2009)

20 Lin, A. E., O'Brien, B., Demmer, L. A., Almeda, K. K., Blanco, C. L., Glasow, P. F. et al. Prenatal features of Costello syndrome: ultrasonographic findings and atrial tachycardia. Prenat. Diagn. 29, 682-690 (2009).

21 Piccione, M., Piro, E., Pomponi, M. G., Matina, F., Pietrobono, R., Candela, E. et al. A premature infant with Costello syndrome due to a rare G13C HRAS mutation. Am. J. Med. Genet. A. 149A, 487-489 (2009).

22 Sol-Church, K., Stabley, D. L., Demmer, L. A., Agbulos, A., Lin, A. E., Smoot, L. et al. Male-to-male transmission of Costello syndrome: G12S HRAS germline mutation inherited from a father with somatic mosaicism. Am. J. Med. Genet. A. 149A, 315-321 (2009).

23 Zhang, H., Ye, J. \& Gu, X. Recurring G12S mutation of HRAS in a Chinese child with Costello syndrome with high alkaline phosphatase level. Biochem. Genet. 47, 868-871 (2009).

24 van der Burgt, I., Kupsky, W., Stassou, S., Nadroo, A., Barroso, C., Diem, A. et al. Myopathy caused by HRAS germline mutations: implications for disturbed myogenic differentiation in the presence of constitutive HRas activation. J. Med. Genet. 44, 459-462 (2007).

25 McGrath, J. P., Capon, D. J., Goeddel, D. V. \& Levinson, A. D. Comparative biochemical properties of normal and activated human ras p21 protein. Nature. 310, 644-649 (1984).

26 Al-Mulla, F., Milner-White, E. J., Going, J. J. \& Birnie, G. D. Structural differences between valine-12 and aspartate-12 Ras proteins may modify carcinoma aggression. J. Pathol. 187, 433-438 (1999).

27 Feig, L. A. \& Cooper, G. M. Relationship among guanine nucleotide exchange, GTP hydrolysis, and transforming potential of mutated ras proteins. Mol. Cell. Biol. 8 , 2472-2478 (1988)

28 Der, C. J., Weissman, B. \& Macdonald, M. J. Altered guanine-nucleotide binding and $\mathrm{H}$-Ras transforming and differentiating activities. Oncogene. 3, 105-112 (1988).

29 Sikora, E., Arendt, T., Bennett, M. \& Narita, M. Impact of cellular senescence signature on ageing research. Ageing Res. Rev. 10, 146-152 (2010).

30 Serrano, M., Lin, A. W., McCurrach, M. E., Beach, D. \& Lowe, S. W. Oncogenic ras provokes premature cell senescence associated with accumulation of p53 and p16INK4a. Cell 88, 593-602 (1997).

31 Narita, M., Nunez, S., Heard, E., Narita, M., Lin, A. W., Hearn, S. A. et al. Rb-mediated heterochromatin formation and silencing of $\mathrm{E} 2 \mathrm{~F}$ target genes during cellular senescence. Cell 113, 703-716 (2003).

32 Di Micco, R., Fumagalli, M., Cicalese, A., Piccinin, S., Gasparini, P., Luise, C. et al. Oncogene-induced senescence is a DNA damage response triggered by DNA hyperreplication. Nature 444, 638-642 (2006).
33 Bartkova, J., Rezaei, N., Liontos, M., Karakaidos, P., Kletsas, D., Issaeva, N. et al. Oncogene-induced senescence is part of the tumorigenesis barrier imposed by DNA damage checkpoints. Nature 444, 633-637 (2006).

34 Narita, M. \& Lowe, S. W. Senescence comes of age. Nat. Med. 11, 920-922 (2005).

35 Campisi, J. Suppressing cancer: the importance of being senescent. Science 309, 886-887 (2005).

36 Kawame, H., Matsui, M., Kurosawa, K., Matsuo, M., Masuno, M., Ohashi, H. et al. Further delineation of the behavioral and neurologic features in Costello syndrome. Am. J. Med. Genet. A. 118A, 8-14 (2003).

37 Kalfa, D., Fraisse, A. \& Kreitmann, B. Medical and surgical perspectives of cardiac hypertrophy in Costello syndrome. Cardiol. Young 19, 644-647 (2009).

38 Digilio, M. C., Sarkozy, A., Capolino, R., Chiarini Testa, M. B., Esposito, G., de Zorzi, A. et al. Costello syndrome: clinical diagnosis in the first year of life. Eur. J. Pediatr. 167, 621-628 (2008).

39 Schuhmacher, A. J., Guerra, C., Sauzeau, V., Canamero, M., Bustelo, X. R. \& Barbacid, M. A mouse model for Costello syndrome reveals an Ang II-mediated hypertensive condition. J. Clin. Invest. 118, 2169-2179 (2008).

40 Kaji, M., Kurokawa, K., Hasegawa, T., Oguro, K., Saito, A., Fukuda, T. et al. A case of Costello syndrome and glycogen storage disease type III. J. Med. Genet. 39, E8 (2002).

41 Omori, I., Shimizu, M. \& Watanabe, T. An infant with Costello syndrome and a rare HRAS mutation (G12C). J. Jpn. Pediatr. Soc. 114, 1592-1597 (2010).

42 Gripp, K. W., Hopkins, E., Doyle, D. \& Dobyns, W.B. High incidence of progressive postnatal cerebellar enlargement in Costello syndrome: brain overgrowth associated with HRAS mutations as the likely cause of structural brain and spinal cord abnormalities. Am. J. Med. Genet. A. 152A, 1161-1168 (2010).

43 Gregersen, N. \& Viljoen, D. Costello syndrome with growth hormone deficiency and hypoglycemia: a new report and review of the endocrine associations. Am. J. Med. Genet. A. 129A, 171-175 (2004).

44 Stein, R. I., Legault, L., Daneman, D., Weksberg, R. \& Hamilton, J. Growth hormone deficiency in Costello syndrome. Am. J. Med. Genet. A. 129A, 166-170 (2004).

45 Alexander, S., Ramadan, D., Alkhayyat, H., Al-Sharkawi, I., Backer, K. C., El-Sabban, F. et al. Costello syndrome and hyperinsulinemic hypoglycemia. Am. J. Med. Genet. A. 139, 227-230 (2005).

46 Santoriello, C., Deflorian, G., Pezzimenti, F., Kawakami, K., Lanfrancone, L., d’Adda di Fagagna, F. et al. Expression of H-RASV12 in a zebrafish model of Costello syndrome causes cellular senescence in adult proliferating cells. Dis. Model. Mech. 2, 56-67 (2009).

47 White, S. M., Graham, J. M. Jr, Kerr, B., Gripp, K., Weksberg, R., Cytrynbaum, C. et al. The adult phenotype in Costello syndrome. Am. J. Med. Genet. A. 136, 128-135 (2005).

48 Seeburg, P. H., Colby, W. W., Capon, D. J., Goeddel, D. V. \& Levinson, A. D. Biological properties of human $\mathrm{C}-\mathrm{Ha}$-ras 1 genes mutated at codon 12 . Nature $312,71-75$ (1984).

49 Fasano, O., Aldrich, T., Tamanoi, F., Taparowsky, E., Furth, M. \& Wigler, M. Analysis of the transforming potential of the human $\mathrm{H}$-ras gene by random mutagenesis. Proc. Natl Acad. Sci. USA 81, 4008-4012 (1984).

50 Jakobisiak, M. \& Golab, J. Statins can modulate effectiveness of antitumor therapeutic modalities. Med. Res. Rev. 30, 102-135 (2010).

51 Li, W., Cui, Y., Kushner, S. A., Brown, R. A., Jentsch, J. D., Frankland, P. W. et al. The HMG-CoA reductase inhibitor lovastatin reverses the learning and attention deficits in a mouse model of neurofibromatosis type 1. Curr. Biol. 15, 1961-1967 (2005).

52 Krab, L. C., de Goede-Bolder, A., Aarsen, F. K., Pluijm, S. M., Bouman, M. J., van der Geest, J. N. et al. Effect of simvastatin on cognitive functioning in children with neurofibromatosis type 1: a randomized controlled trial. JAMA 300, 287-294 (2008).

53 Dale, K. M., Coleman, C. I., Henyan, N. N., Kluger, J. \& Whitem, C. M. Statins and cancer risk: a meta-analysis. JAMA 295, 74-80 (2006). 